\title{
Effects of Magnolol on Impairment of Learning and Memory Abilities Induced by Scopolamine in Mice
}

\author{
Yang-si Li, ${ }^{a}$ Ying-fen Hong, ${ }^{a}$ Jiang He, ${ }^{a}$ Jian-xun Lin, ${ }^{a}$ Yi-long Shan, ${ }^{a}$ Dong-ying Fu, ${ }^{a}$ \\ Zhi-peng Chen, ${ }^{a}$ Xin-ran Ren, ${ }^{b}$ Zhi-hong Song, ${ }^{* c}$ and Liang Tao ${ }^{c}$ \\ ${ }^{a}$ Clinical Medicine Department, Sun Yet-Sen University; ${ }^{c}$ Zhongshan School of Medicine, Sun Yet-Sen University; \\ 74 Zhongshan 2nd Road, Guangzhou 510089, China: and ${ }^{b}$ School of International Studies, Sun Yet-Sen University; \\ Guangzhou 510275, China. \\ Received October 6, 2012; accepted February 5, 2013; advance publication released online February 28, 2013
}

\begin{abstract}
Alzheimer's disease (AD), one of the most common forms of dementia, is primarily ascribed to the cholinergic deficits and neuronal dysfunction. Magnolol (Mag), a bioactivator extracted from Magnolia officinalis, has protective effects on cholinergic neurons, but the specific mechanism remains unknown. To further evaluate the therapeutic effects of Mag on the learning and memory impairment in a scopolamine (Scop)-induced mouse model, the passive avoidance and the Morris water maze tests, the measurement of the ratio of brain/hippocampus to body weight, activities of acetyl cholinesterase (AChE), superoxide dismutase (SOD), total nitric oxide synthase (total NOS) and the content of methane dicarboxylic aldehyde (MDA) in hippocampus homogenate as well as the immunefluorescence staining of the AChE positive nerve fibers were performed. Therapeutically treated with Mag, the impaired abilities of learning and memory of the Scop-induced mice were almost restored to the native levels. The restored AChE, total NOS and SOD activities and the MDA level were observed, with a relatively normal density of $\mathrm{AChE}$ positive nerve fibers in hippocampus CA3 molecular layer. The improving efficacy of Mag on learning and memory impairment induced by Scop is dose-dependent, indicating that Mag has potential neuroprotective effects against neuronal impairment and memory dysfunction induced by Scop in mice. The underlying mechanisms may be associated with the anti-oxidative effects of Mag and its protective effects on hippocampus cholinergic neurons.
\end{abstract}

Key words magnolol; learning ability; memory ability; anti-oxidation; scopolamine

Alzheimer's disease (AD), characterized by a progressive decline in cognitive function and memory, is one of the most common forms of dementia in the aging population. ${ }^{1)}$ In terms of the neuropathological features of memory loss and cognitive dysfunction, cholinergic deficits and neuronal dysfunction have been considered to be the primary causes as observed in patients with $\mathrm{AD}^{2)}$ Accumulating documents show that cholinergic injury of hippocampus neurons is the root cause of weakened function of learning and memory. ${ }^{3)}$ In addition, total nitric oxide synthase (NOS) level in hippocampus also plays a core role in the mice's learning and memory. ${ }^{4)}$ Thus, deeper understanding the cause of hippocampus neurons injury is very important for the prevention and treatment of learning and memory decline or AD. Since it has been confirmed that dysfunction in cholinergic system triggers the weakening of learning and memory functions, and changes in the activity of acetylcholineasterase (AChE) have been attracted much attention. $^{5-7)}$

Magnolol (Mag) is a bioactivator extracted from Magnolia officinalis, a traditional Chinese medicine, and contains phenolic hydroxyl that is lipid-soluble to shuttle through the bloodbrain barrier. ${ }^{8)}$ Pharmacologically, Mag has various activities in anti-inflammatory, anti-bacterial, anti-allergic effects. ${ }^{8,9)}$ The in-vitro laboratory experiments have confirmed that Mag can increase acetylcholine (ACh) in hippocampus area of normal mice and the mice with cholinergic deficiencies, showing its protective effects on cholinergic neurons. ${ }^{10,11)}$ Mag can also promote neurons' growth in embryonic hippocampus and cortex directly or indirectly. ${ }^{12)}$ However, the specific mechanism remains unknown. In this study, we investigated the effects

The authors declare no conflict of interest. of Mag on scopolamine (Scop)-induced learning and memory impairment in mice to explore the underlying mechanisms.

\section{MATERIALS AND METHODS}

Materials Mag $\left\{6,6^{\prime}, 7,12\right.$-tetramethoxy-2,2'-dimethyl-1beta-berbaman\} (Fig. 1, purity $>99 \%$ ), Scop and donepezil were purchased from Guangdong Institute for Drug Control (Guangzhou, China). Mag was dissolved with propylene glycol, while Scop and donepezil with normal saline (NS); mouse anti-NeuN (1:500, Novus, U.S.A.); rabbit anti-AChE $(1: 100$, Novus, U.S.A.); anti-mouse immunoglobulin G (IgG) $(1: 200$, Sigma, St. Louis, MO, U.S.A.); tetraethylrhodamine isothiocyanate conjugated anti-rabbit IgG (1:400, Sigma); All other reagents were from Guangzhou Chemical Reagent Factory (Guangzhou, China), unless otherwise indicated.

Animals Mice were maintained in a temperature-controlled room, with humidity of $55 \pm 5 \%$ and a light/dark cycle of $12 / 12 \mathrm{~h}$ (lights on at 8 a.m.). Sixty adult male Kunming mice weighing $18-22 \mathrm{~g}$ were used to evaluate various characters and behaviors, of which 48 mice were allocated for the biomedical assay, and another 12 for the double-staining immunofluorescence of $\mathrm{AChE}$ positive fibers in mice brain. All

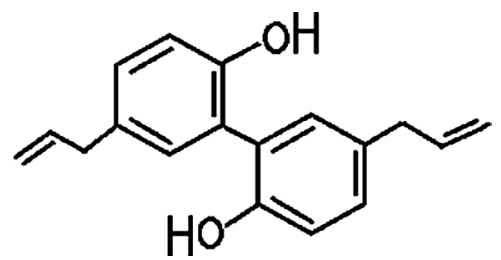

Fig. 1. Chemical Structure of Mag 


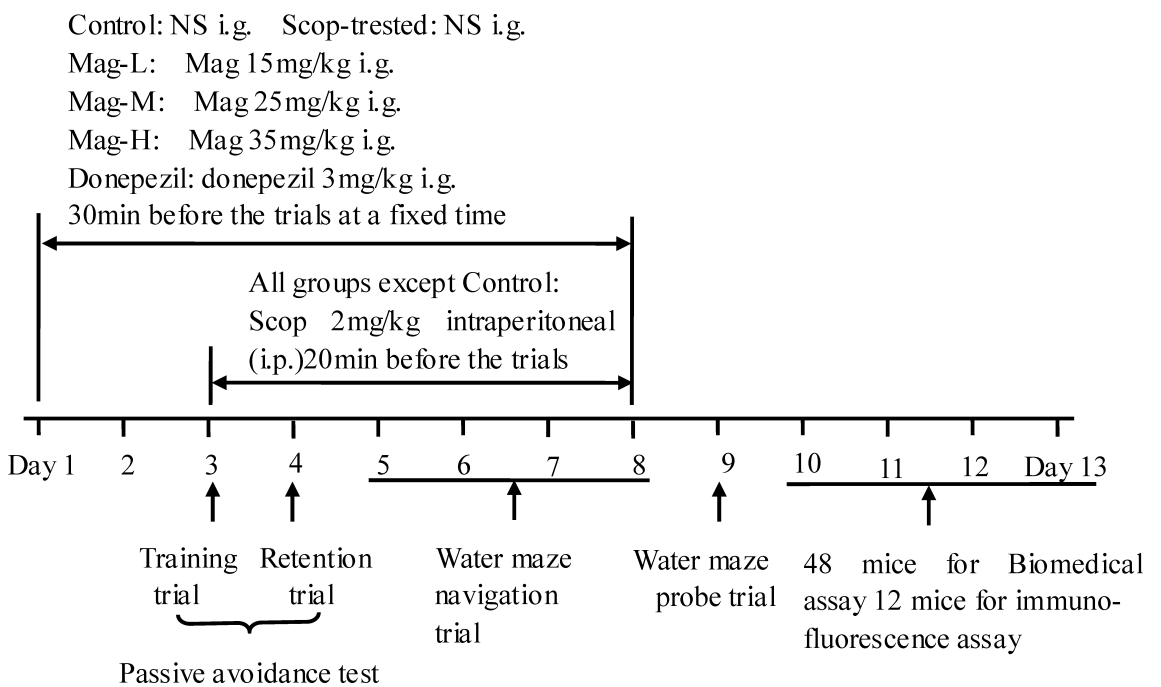

Fig. 2. The Schedule of Drug Administrations

Mag was administered daily for $2 \mathrm{~d}$ before any trail. On the day of the behavioral test, Mag and Scop were successively administered before the trials. Passive avoidance test were performed on the third and fourth day while Morris water maze test were on the fifth to ninth days. Twenty-four hours after the behavioral test, 48 mice were used for the biomedical assay and 12 mice for immunofluorescence assay.

animal experiments were conducted in accordance with the guidelines and regulations of Sun Yet-Sen University Animal Care and Use Committee.

Drug Administrations All mice were given the intragastric (i.g.) administration of the indicated dose of medication at the fixed time point in the morning for 8 continuous days, according to the schedule shown in Fig. 2. From the third day to the eighth day, except mice in the control group treated with NS, Scop $(2 \mathrm{mg} / \mathrm{kg}$ ) was intraperitoneally (i.p.) injected into the mice $20 \mathrm{~min}$ before training.

Passive Avoidance Test The passive avoidance response was determined using a "step-through" apparatus that consists of an illuminated and a dark compartment (each $20.3 \times 15.9 \times 21.3 \mathrm{~cm}$ in size) adjoining each other through a small gate with a grid floor consisting of $3.175 \mathrm{~mm}$ stainless steel rod set $8 \mathrm{~mm}$ apart. On the training trial (on the third day), the mice were placed in the illuminated compartment facing away from the dark compartment. When the mice moved completely into the dark compartment; they would receive an electric shock ( $1 \mathrm{~mA}, 3 \mathrm{~s}$ duration). Then, the mice would return to their home cage. Twenty-four hours after the training trial (on the fourth day), the mice were placed in the illuminated compartment and the latency period to firstly enter the dark compartment was defined as "retention" and described as "step-through latency." The entrance number of a mouse walking into the dark compartment in $5 \mathrm{~min}$ was described as "error times." "Step-through latency" and "error times" were recorded by the "step-through" apparatus automatically. Scop $(2 \mathrm{mg} / \mathrm{kg})$ was i.p. into mice $20 \mathrm{~min}$ before the trial.

Morris Water Maze Test Morris water maze test was carried out with the particular equipment and processed with SMART-CS program. A round plastic pool $(35 \mathrm{~cm}$ in height, $100 \mathrm{~cm}$ in diameter) was filled with milky water and kept at $22-25^{\circ} \mathrm{C}$. An escape platform $(14.5 \mathrm{~cm}$ in height, $4.5 \mathrm{~cm}$ in diameter) is submerged $0.5-1 \mathrm{~cm}$ below the surface of the water in quadrant III. During the navigation test (on the fifth day to the eighth day), the mice were placed in a pool of water and allowed to search for and stay on the platform for $10 \mathrm{~s}$, and then returned to the home cage. The mice that did not find the platform within $60 \mathrm{~s}$ were placed on the platform for $10 \mathrm{~s}$. On the spatial probe trial, $24 \mathrm{~h}$ later after the last navigation practice (4 times/d for $4 \mathrm{~d}$ ), the mice were given the testing assay. They were allowed to swim for one minute. A camera connected to the SMART-LD program was placed above the center of the pool to monitor the swimming pattern of the mice. The escape latency (the time mice took to first cross the platform), cross times (the total times of mice cross the platform in one minute) and swimming pattern of each mouse were automatically recorded by the program. Scop $(2 \mathrm{mg} / \mathrm{kg}$, i.p.) was injected into mice $20 \mathrm{~min}$ before the trials.

Ratio of Brain/Hippocampus to Body Weight Twentyfour hours after the behavioral test, the mice were quickly decapitated and the brains were carefully dissected. The blood of brains was washed away with the iced NS and absorbed the remaining NS with filter paper, and then the brains were weighed to calculate the ratios of brain to body weight. Similarly, the hippocampus were separated on an iced operating floor and weighed to calculate the ratios of hippocampus to body weight, following rinsing with the cooled NS.

Determination of the Activities of AChE, Total NOS, Superoxide Dismutase (SOD) and Methane Dicarboxylic Aldehyde (MDA) Level The hippocampus were placed in the precooled homogenate medium (sterile NS) by weight/volume ratio of $1: 9$ to make $10 \%$ tissue homogenate, and then centrifuged at a low temperature at $15000 \mathrm{rpm} / \mathrm{min}$ for $2 \mathrm{~min}$. The concentration of the total protein (TP) was determined and the activities of enzymes AChE, total NOS, SOD and MDA level were quantified by using the corresponding detection kits (Nanjing Jiancheng Bioengineering Institute, Nanjing, China), according to the manufacturer's instructions. The absorbance was read respectively at $595 \mathrm{~nm}, 412 \mathrm{~nm}, 530 \mathrm{~nm}, 550 \mathrm{~nm}$ and $532 \mathrm{~nm}$ using 752S UV-visible spectrophotometer (LingGuang, ShangHai, China). TP level is expressed as $\mathrm{g} / \mathrm{L}$, and the rest indexes are expressed as $\mathrm{U} / \mathrm{mg}$ proteins (prot).

Immunofluorescence Staining of $\mathrm{AChE}$ Positive Nerve Fibers After the anesthesia with 10\% chloralic hydras, mice were transcardially perfused with $4 \%$ paraformaldehyde (PFA) 
after rinsing with $0.01 \mathrm{M}$ phosphate buffered saline (PBS). Brains of the mice were carefully dissected and fixed in $4 \%$ PFA at $4^{\circ} \mathrm{C}$ overnight, then transferred to $30 \%$ sucrose in $0.01 \mathrm{M}$ PBS at $4^{\circ} \mathrm{C}$ overnight again. Brains were embedded in OCT Compound (Tissue-Tek) and stored at $-80^{\circ} \mathrm{C}$ for later sectioning. Coronal sections were sliced at thickness of $35 \mu \mathrm{m}$ using freezing microtome (LEICA, Germany). The sections were blocked in $2 \%$ BSA with $0.3 \%$ Triton X-100 in $0.01 \mathrm{M}$ PBS at room temperature for $30 \mathrm{~min}$, and then incubated in mouse anti-NeuN (1:500, Novus) and rabbit anti-AChE ( $1: 100$, Novus) primary antibodies together for $72 \mathrm{~h}$ at $4{ }^{\circ} \mathrm{C}$. After washing with PBS, the sections were incubated with fluorescein isothiocyanate (FITC)-conjugated anti-mouse IgG $(1: 200$, Sigma) and TRITC-conjugated anti-rabbit $\operatorname{IgG}(1: 400$, Sigma) at room temperature for $2 \mathrm{~h}$ in the dark. After washing in PBS, the sections were mounted with anti-fade mounting media ( $50 \%$ glycerin in $0.5 \mathrm{M}$ buffer bicarbonate, $\mathrm{pH} 9.5)$, and observed under fluorescence microscopy (Zeiss, U.S.A.).

Data Analysis The data are presented as mean \pm standard error (S.E.), analyzed using SPSS 13.0 software. The homogeneity of variances was assessed with a Bartlett test. If variances were homogeneous, differences between groups and treatment were assessed by one-way or two-way analysis of variance (ANOVA). One-way ANOVA was used to analyze data for the activities of AChE, total NOS, and SOD and the levels of MDA. The data obtained from Morris water maze (escape latency, cross times and percent time and course of quadrant III) and passive avoidance test, step-through latency and error times) were analyzed using two-way ANOVA, followed by a post-hoc test. When variances were not homogeneous, the Kruskal-Wallis test was used to assess differences between groups for nonparametric analyses. If the $p$-value in the Kruskal-Wallis test was significant, the differences between pairs of means were assessed by Fisher's protected least significant difference (PLSD) post hoc test. A value of $p<0.05$ was considered to be statistically significant.

\section{RESULTS}

Effects of the Mag on Animals Characteristics The ratio of brain to body weight and the ratio of hippocampus to body weight are indicators to reflect the intelligence of mice. In comparison to the control group, no obvious changes were observed in body weight of the mice in the Scop-treated group $24 \mathrm{~h}$ after the last feed $(p>0.05)$, while the ratio of brain to body weight and the ratio of hippocampus to body weight were significantly lowered $(p<0.05)$ (Table 1$)$, indicating that the brains, particularly the hippocampuses of Scop-treated mice may atrophy.

Table 1. The Ratio of Brain/Hippocampus to Body Weight

\begin{tabular}{llll}
\hline \hline Group & $\begin{array}{c}\text { Body } \\
\text { weight }(\mathrm{g})\end{array}$ & $\begin{array}{c}\text { Ratio of brain to } \\
\text { body weight }(\mathrm{g} / \mathrm{g})\end{array}$ & $\begin{array}{c}\text { Ratio of hippo- } \\
\text { campus to body } \\
\text { weight }(\mathrm{g} / \mathrm{g})\end{array}$ \\
\hline $\begin{array}{l}\text { Control } \\
\text { Scop-treated }\end{array}$ & $\begin{array}{l}28.8167 \pm 1.5145 \\
29.9167 \pm 2.1245\end{array}$ & $0.0129 \pm 0.0014$ & $0.0013 \pm 0.0001$ \\
\hline $\begin{array}{l}\text { Twenty-four hours after the behavioral test, mice were quickly decapitated, then } \\
\text { the brains and the hippocampuses were separated. The washed brains and hippocam- }\end{array}$ \\
puses were weighed respectively to calculate the ratios of brain and hippocampus to \\
body weight. The data presented are shown as the mean \pm S.E., $n=6 . * p<0.05$ vs. the \\
control group.
\end{tabular}

Effects of the Mag on Passive Avoidance Test Retention latency in the passive avoidance test are known to indicate long-term memory functions in rodents. ${ }^{13)}$ The step-through latency of the mice in the Scop-treated group within $5 \mathrm{~min}$ significantly decreased in comparison with the control group $(p<0.05)$, while the error times significantly increased $(p<0.05)$, indicating that Scop induced memory impairment. In another way, compared to the Scop-treated group, the stepthrough latency of mice in the Mag-H treated group and the donepezil-treated group within $5 \mathrm{~min}$ significantly increased $(p<0.05)$, and the corresponding error times in the Mag-M treated group, the Mag-H treated group and donepezil-treated group reduced $(p<0.05)$. There was no significant difference between any Mag-treated group and the donepezil-treated group (Table 2). These data suggested that Mag ameliorated impairment of long-term memory.

Effects of the Mag on Morris Water Maze Test The effect of Mag on the hippocampus-dependent spatial learning ability and long-term spatial memory was evaluated using the Morris water maze test. ${ }^{14)}$ In the spatial probe trial, the escape latency of the mice in the Scop-treated group significantly increased in comparison to the control group $(p<0.01)$, while the times of crossing the platform area decreased $(p<0.05)$, which prove the successfully constructed Scop-induced model mice. Compared to mice in the Scop-treated group, the time for mice in the Mag-M, Mag-H and donepezil-treated groups to first arrive to the platform were significantly reduced $(p<0.05)$, and the times of crossing the platform area were significantly increased $(p<0.05)$. These data suggest that Mag augmented spatial learning ability in Scop-induced mice. No significant difference was observed in mice among the two Mag-treated and donepezil-treated groups (Figs. 3A, B).

In the spatial probe trial, the activity time and moving distances of the mice of the Mag-M treated group and the donepezil treated group in quadrant III (where the platform was placed) were significantly higher than the mice of the Scop-treated group $(p<0.05)$, and the responding time also improved in the Mag-L treated group $(p<0.05)$ (Fig. 3C). The data suggest that the effect of Mag to ameliorate the impaired abilities of learning and memory induced by Scop is dependent on the Mag dose.

Meanwhile, with the training time (4 times/d for $4 \mathrm{~d}$ ) increasing, the escape latency of the mice in the Scop-treated group maintained at a high level, while that of the control group was significantly shortened $(p<0.05)$, indicating that Scop caused spatial memory impairment of mice. Compared

Table 2. The Step-through Latency and the Error Times in the Passive Avoidance Test

\begin{tabular}{lcc}
\hline \hline Group & Step-through latency $(\mathrm{s})$ & Error times \\
\hline Control & $286.67 \pm 28.13$ & $0.25 \pm 0.46$ \\
Scop-treated & $132.72 \pm 70.44^{*}$ & $1.25 \pm 0.46^{*}$ \\
Mag-L & $160.74 \pm 93.25^{*}$ & $0.75 \pm 0.71$ \\
Mag-M & $181.00 \pm 76.83^{*}$ & $0.50 \pm 0.53^{* *}$ \\
Mag-H & $210.25 \pm 60.63^{* * * *}$ & $0.625 \pm 0.52^{* *}$ \\
Donepezil & $230.41 \pm 66.77^{* *}$ & $0.50 \pm 0.53^{* *}$ \\
\hline
\end{tabular}

Scop $(2 \mathrm{mg} / \mathrm{kg})$ was injected i.p. into mice $20 \mathrm{~min}$ before the passive avoidance test. Twenty-four hours later after the training trail, the step-through latency of the mice and the error times within $5 \mathrm{~min}$ were measured. The data presented are shown as the mean \pm S.E., $n=8$. ${ }^{*} p<0.05$ vs. the control group ; $* * p<0.05 v s$. the Scoptreated group. 
A

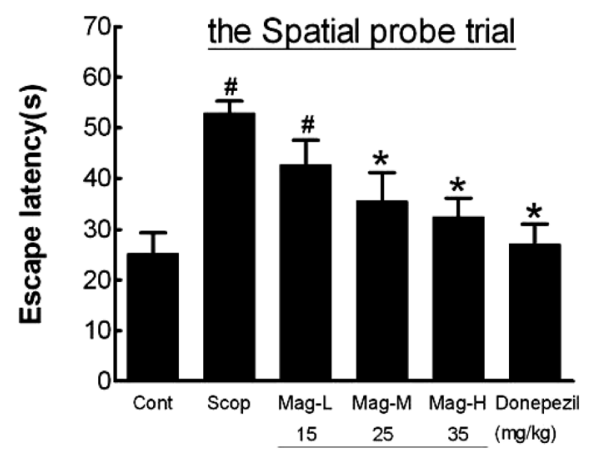

B

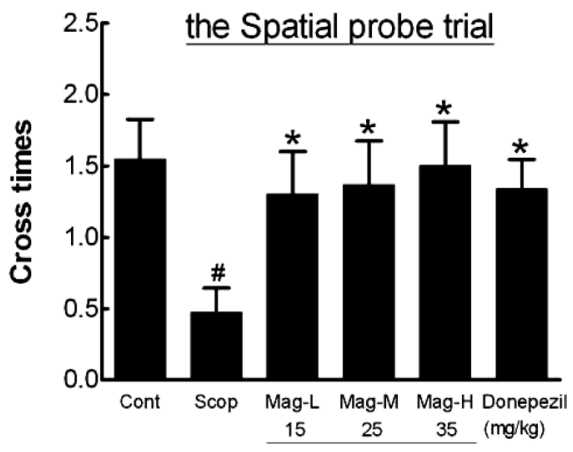

percent course of Quadrant III

$\square$ percent time of Quadrant III

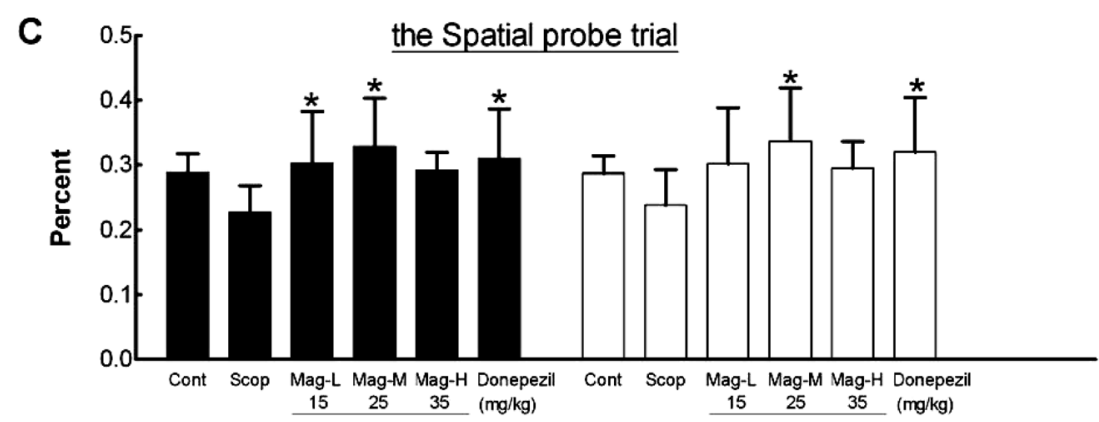

D

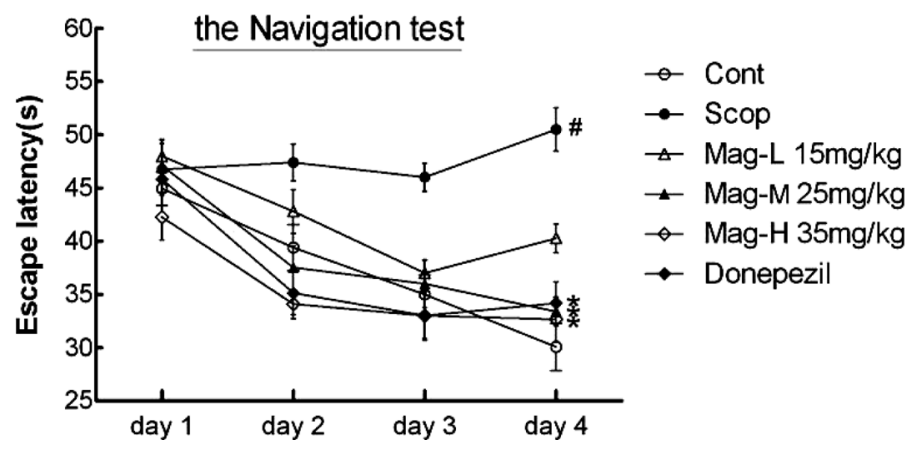

Fig. 3. Protective Effect of Mag on the Memory Impairment Induced by Scop on Morris Water Maze Test

A and B Scop $(2 \mathrm{mg} / \mathrm{kg})$ was i.p. injected into the mice $20 \mathrm{~min}$ immediately before the first trial on each day. Twenty-four hours later after the final navigation trial (4 times/d for $4 \mathrm{~d}$ ); the mice were given the spatial probe test. (A) The escape latency of mice for the spatial probe test was measured. (B) The times of crossing the platform area were measured. (C) In the spatial probe trial, the activity time and moving distances of the mice in quadrant III (where the platform was placed) were measured. (D) The escape latency of the mice was measured for four consecutive days (the navigation test). All data presented are shown as the mean \pm S.E. $\left(n=8 /\right.$ group). ${ }^{\#} p<0.05 v s$. the control group, $* p<0.05$ vs. the Scop-treated group.

to mice in the Scop-treated group, the escape latency of the mice in the Mag-M, Mag-H and donepezil-treated groups were significantly shortened with the increase in training time $(p<0.05)$, suggesting a preventive effect of Mag on Scopinduced memory impairment (Fig. 3D).

Effects of the Mag on the Activity of AChE ACh is an important neurotransmitter related to learning and memory; however, it is difficult to measure the content of $\mathrm{ACh}$ as it decomposes rapidly. It is reliable to indicate the distribution of cholinergic nerves in the hippocampus with the activity of $\mathrm{AChE}^{15)}$ and the density of $\mathrm{AChE}$ positive nerve fibers. The result showed that the activity of $\mathrm{AChE}$ in hippocampus of the mice in the Scop-treated group and the donepezil-treated group decreased compared to the control group $(p<0.05)$, indicating that the dysfunction of central cholinergic nerves in the process of brain atrophy. However, the AChE inhibitor donepezil did improve the impaired learning and memory abilities in both passive avoidance test and Morris water maze test. The reason may be its improving effect of anti-oxidation and blood flow increasing, ${ }^{16)}$ which eased the degeneration of AChE neuron. Compared to mice in the Scop-treated group, the AChE activity in the Mag-H treated group was restored $(p<0.05)$, nearly to the normal level (compared to the control group, $p>0.05$ ), indicating Mag may have the effect to reverse the dysregulation of central cholinergic nerves combined with the improving in behavior tests. The AChE activity in the Mag-M treated group was also significantly improved, compared to the Mag-L treated group $(p<0.05)$ (Table 3).

Effects of the Mag on the Activity of Total NOS It has reported that the decline of total NOS activity in the hip- 
Table 3. The Activity of AChE of Hippocampus

\begin{tabular}{ll}
\hline \hline Group & \multicolumn{1}{c}{ AChE (U/mg prot) } \\
\hline Control & $0.3354 \pm 0.0650$ \\
Scop-treated & $0.2511 \pm 0.0478 *$ \\
Mag-L & $0.2019 \pm 0.0742 *$ \\
Mag-M & $0.2774 \pm 0.0283 * * *$ \\
Mag-H & $0.3165 \pm 0.0369 * * * * *$ \\
Donepezil & $0.2552 \pm 0.0671 *$ \\
\hline
\end{tabular}

The hippocampus was separated and lyzed, then take the supernatant of the centrifuged tissue homogenate for the determination of $\mathrm{AChE}$ according to the instructions of the determination kit. The data presented are shown as the mean \pm S.E., $n=6$. ${ }^{*} p<0.05 v s$. the control group; ${ }^{* *} p<0.05$ vs. the Scop-treated group; $* * * p<0.05 v s$. the Mag-L treated group.

pocampus may be one of the main reasons that caused Scopinduced amnesia, and the maintenance of it contributed to the improvement of learning and memory. ${ }^{17-19)}$ Meanwhile, the activity of total NOS in the hippocampus is one of the classical indexes to the evaluation of the anti-oxidation effects. Results in Table 4 showed that the total NOS activity in hippocampus of mice in the Scop-treated group was significantly lower than that in the control group $(p<0.05)$, indicating the impairment of memory. In addition, the total NOS activity in the Mag-L/M/H treated groups and the donepezil-treated group significantly restored, compared to the Scop-treated group $(p<0.05)$, indicating that Mag and donepezil can restore the Scop-induced decrease in total NOS activity. The effects of all 3 doses of Mag on total NOS activity were stronger than donepezil $(p<0.05)$. The effect of high dose of Mag on total NOS activity was more potent than the low and median dose of Mag $(p<0.05)$. This result suggests that Mag protects the hippocampus NOS neurons and reverses memory impairment induced by Scop. It can be inferred that Mag increases NO content by elevating total NOS activity to improve learning and memory abilities.

Effects of the Mag on the Activity of SOD and the Content of MDA The development of hippocampus neurons degeneration is closely related to oxidative stress. ${ }^{20)}$ The activity of SOD and the content of MDA in the hippocampus are the classical indexes to evaluate the anti-oxidative effects. From the results in Table 5, we observed that the content of MDA in hippocampus of the mice in the Scop-treated group significantly increased and the SOD activity decreased compared to the control group $(p<0.05)$. Treatment with all 3 doses of Mag and donepezil decreased the MDA content and restored the SOD activity $(p<0.05)$. The effect of high dose of Mag was more potent than that of low and median dose of Mag $(p<0.05)$. The results indicate that Mag may be able to scavenge oxygen free radicals and increase the oxidation resisting capacity of the hippocampus, therefore preventing the hippocampus neurons from lipid peroxidation damage.

Protective Effects of Mag on Recovery of the AChE Positive Nerve Fibers To explore the changes of AChE with Mag administration, immuno-fluorescent staining of AChE were conducted. The density of AChE positive nerve fibers located in hippocampus CA3 molecular layer significantly decreased in the Scop-treated mice compared with the control group (Figs. 4B versus 4A), further proving the degeneration of AChE neurons. With Mag treatment, the density of AChE positive nerve fibers in the Mag-M treated group was almost reversed to the native status as is shown in the control group
Table 4. The Activity of Total NOS of Hippocampus

\begin{tabular}{lll}
\hline \hline Group & $n$ & \multicolumn{1}{c}{ Total NOS (U/mg prot) } \\
\hline Control & 5 & $66.1900 \pm 9.9740$ \\
Scop-treated & 6 & $25.8800 \pm 9.5400^{*}$ \\
Mag-L & 6 & $53.8983 \pm 9.5547 *, * *$ \\
Mag-M & 6 & $62.5417 \pm 9.2133 * *$ \\
Mag-H & 5 & $75.1020 \pm 10.8812 * *, * * *, * * * * * * * * *$ \\
Donepezil & 6 & $36.7067 \pm 1.9379 *, * * * * *, * * * *$ \\
\hline
\end{tabular}

The hippocampus was separated and lyzed, then take the supernatant of the centrifuged tissue homogenate for the determination of total NOS according to the instructions of the determination kit .The data presented are shown as the mean \pm S.E., the number is indicated as ' $n$.' $* p<0.05$ vs. the control group; ${ }^{*} p<0.05 v s$. the Scoptreated group; $* * * p<0.05$ vs. the Mag-L treated group; $* * * * p<0.05$ vs. the Mag-M treated group. $* * * * * p<0.05 v s$. the donepezil-treated group.

Table 5. The Contents of MDA, Activity of SOD of Hippocampus

\begin{tabular}{lccc}
\hline \hline Group & $n$ & $\begin{array}{c}\text { MDA } \\
\text { (nmol/mg prot) }\end{array}$ & SOD (U/mg prot) \\
\hline Control & 5 & $1.3980 \pm 0.7947$ & $9.0749 \pm 0.7409$ \\
Scop-treated & 6 & $2.8517 \pm 0.9807^{*}$ & $6.3965 \pm 0.9553^{*}$ \\
Mag-L & 6 & $0.9033 \pm 0.2177 * *$ & $5.9773 \pm 0.7050^{*}$ \\
Mag-M & 6 & $0.9775 \pm 0.2949 * *$ & $9.2112 \pm 0.5614 * *, * * *$ \\
Mag-H & 5 & $1.1600 \pm 0.2228^{* *}$ & $12.7257 \pm 3.9422^{* * * * * * *, * * * *}$ \\
D & 6 & $1.1767 \pm 0.3354 * *$ & $8.1518 \pm 0.7765$ \\
\hline
\end{tabular}

The hippocampus was separated and lyzed, then take the supernatant of the centrifuged tissue homogenate for the determination of MDA and SOD according to the instructions of the determination kit. The data presented are shown as the mean \pm S.E.; the number is indicated as ' $n$.' $* p<0.05 v s$. the control group; $* * p<0.05 v s$. the Scop-treated group; $* * * p<0.05$ vs. the Mag-L treated group; $* * * * p<0.05$ vs. the Mag-M treated group.

(Figs. 4C versus 4A). The results are consistent with those of biomedical assay, indicating that Mag protects the AChE activity from decreasing and results in the improvement of the impaired abilities of learning and memory.

\section{DISCUSSION}

The neuropathology in AD patients is characterized by a progressive loss of memory and cognitive ability. The central cholinergic system is the primary pathway of learning and memory abilities formation, ${ }^{2}$ and the deficiency in cholinergic system is probably the most common pathogenesis of dementia illnesses such as AD. ${ }^{1)}$

$\mathrm{ACh}$ is an important neurotransmitter related to learning and memory. The cholinergic nerves regulate the excitation and inhibition of the cerebral cortex, influencing the ability of learning and memory. Although using AChE for a marker of cholinergic nerves has limited specificity, it is reliable to indicate the distribution of cholinergic nerves in the hippocampus with the density of AChE positive nerve fibers. ${ }^{15)}$ Therefore, the reduction of the density of $\mathrm{AChE}$ positive nerve fibers indicates the dysfunction of central cholinergic nerves in the process of brain atrophy.

The studies have confirmed that learning and memory impairment goes along with the loss of cholinergic fibers in most cases. $^{21,22)}$ Cholinergic neuron damage in hippocampus, cerebral cortex and other parts of brain can result in cholinergic dysfunction like $\mathrm{AD}$ or other senile dementia. A variety of factors cause brain injury by reducing the density of choline acetyltransferase (ChAT) cells and AChE positive 

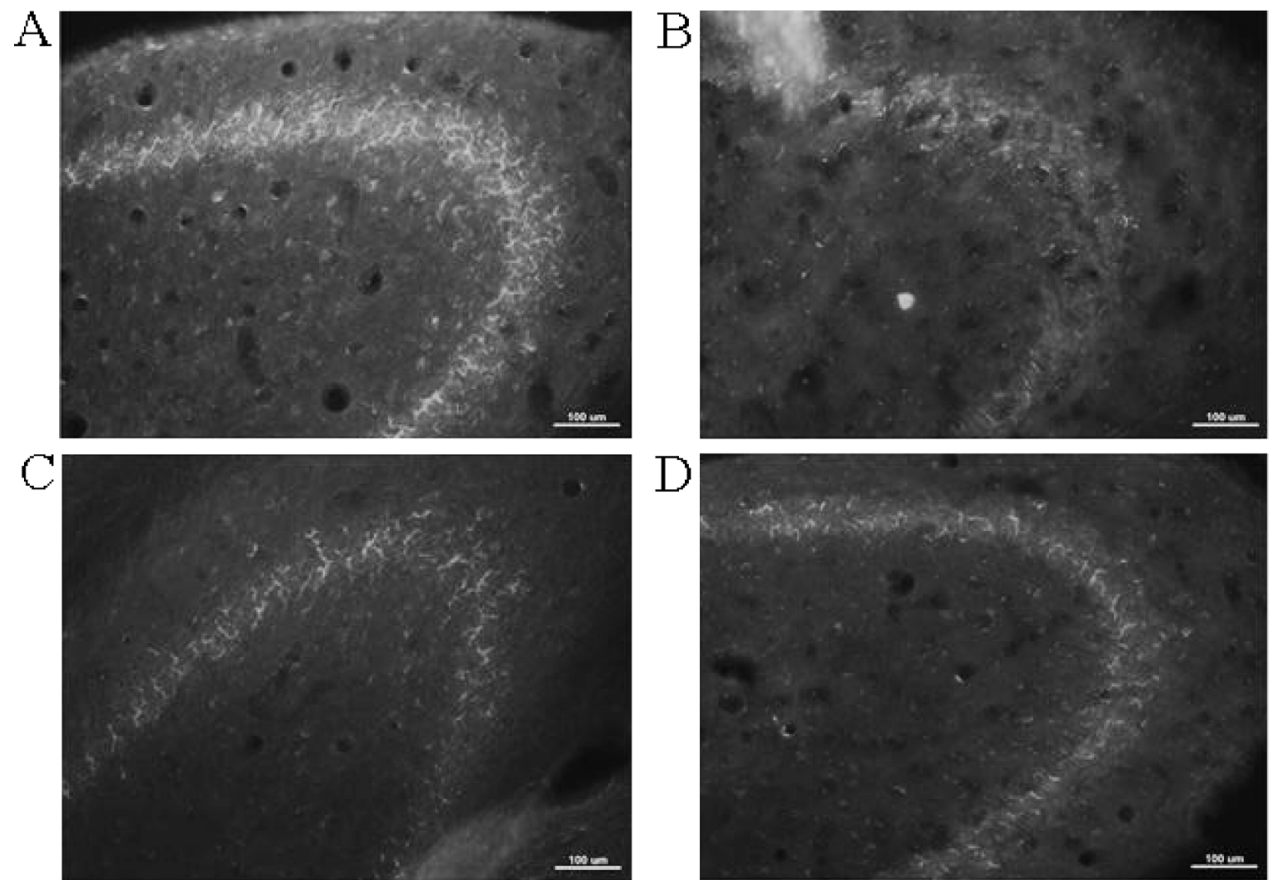

Fig. 4. Protective Function of Mag in Recovery of the AChE Positive Nerve Fibers

AChE positive nerve fibers in hippocampus CA3 molecular layer of experimental mice in the control group (A), in the Scop-treated Group (B), in the Mag-M group (C), and in the donepezil-treated group (D). All pictures are photographed under fluorescent microscope. The scale bar is indicated.

nerve fibers (in particular, hippocampus AChE fiber) in brain as well as by affecting the synthesis, release, uptake of ACh, resulting in brain atrophy and dysfunction of central cholinergic neurons, which may lead to memory and cognitive dysfunction and even the development of dementia. ${ }^{5-7)}$ The decrease of AChE activity indicates the abnormality of central cholinergic neurons, which is often present in the process of brain atrophy. ${ }^{6}$ Moreover, it has been reported that activity of AChE in the brain decreases in some AD patients. ${ }^{23)}$ This may be one of the important reasons why treatments with AChE inhibitors are ineffective in some AD patients. Therefore, protecting central cholinergic neurons from functional degenerative disorders and maintaining the activity of AChE in the hippocampus neurons may be useful for the prevention of the development or progression of $\mathrm{AD}$ and/or other chronic brain degenerative diseases. ${ }^{6}$

Scop has been used in neuro-psychopharmacology as a standard drug that leads to cognitive deficits during the aging process in healthy humans and animals. ${ }^{24)}$ In the present study, intraperitoneal administration of Scop can block the binding sites of $\mathrm{ACh}$ receptor in the cerebral cortex and result in excessive release of $\mathrm{ACh}$, thereby increasing the damage of hippocampus nerves, which can be indicated by the lowered density of AChE positive nerve fibers in hippocampus CA3 molecular layer and the decrease of activity of AChE. Scop induces dysregulation of the cholinergic neuronal pathway and memory circuits in the central nervous system (CNS), resulting in serious impairment in learning, acquisition, and shortterm retention of spatial memory tasks.

In this study, the ratio of brain/hippocampus to body weight decreased in the Scop-treated group, suggesting that the mice's brains, particularly the hippocampuses may atrophy. Moreover, the hippocampus AChE activity significantly decreased in mice of the Scop-treated group, indicating that hippocampus atrophy might reduce AChE fiber density and cause cholinergic neuronal degeneration. Passive avoidance test showed that the step-through latency of mice in $5 \mathrm{~min}$ was significantly shortened and the error times within $5 \mathrm{~min}$ increased, and Morris water maze test showed that the time to first arrive at the platform within $60 \mathrm{~s}$ increased and the times of mice crossing the platform area and the percentage of activity time and distance in the quadrant III (the platform region) were significantly reduced in the Scop-treated group. Meanwhile, with the training time increasing, the search time changed little and maintained at a high level, indicating learning abilities and memory had been damaged, and deficits of cognition and spatial memory had been induced. The treatment of Mag reversed the decrease of AChE activity and deficits of cognition and spatial memory induced by Scop in a dose-dependent manner as well as improved the abilities of learning and memory. These results showed that Mag reverses or resists the memory impairment induced by Scop and these effects may be closely related to the maintenance of $\mathrm{AChE}$ positive nerve fibers in hippocampus.

Knox et al. have reported that the total NOS activity in the hippocampus and prefrontal cortex decreased in Scop-induced mice, ${ }^{24)}$ which is consistent with our study. Besides, repeated administration of total NOS inhibitor, $\mathrm{L}-N^{\mathrm{G}}$-nitro arginine methyl ester (L-NAME), exerts a dual effect on rats' recognition memory abilities, L-NAME disrupted rats' performance in this recognition memory paradigm as compared to that expressed by their vehicle-treated cohorts. ${ }^{17)}$ According to the latest report, Agmatine, decarboxylated arginine, regulates the production of $\mathrm{NO}$, and other metabolites of L-arginine like total NOS to modulate the behavioral function. Intraperitoneal administration of agmatine significantly improved spatial working memory and objects recognition memory in aged rats and restored endothelial total NOS protein to the normal level. ${ }^{18)}$ Epigallocatechin-3-gallate (EGCG) could ameliorate learning and memory deficits in diabetic rats via modulation 
of total NOS activity. ${ }^{19)}$ These results indicated that NOS neurons in hippocampus play an important role in learning and memory. In this study, we demonstrated that Mag recovered total NOS activity induced by Scop nearly to the normal level. This result suggested that Mag protects the hippocampus NOS neurons and reverses memory impairment induced by Scop. It can be inferred that Mag increases NO content by elevating total NOS activity to improve learning and memory abilities.

The development of hippocampus neurons degeneration is closely related to oxidative stress. ${ }^{20)}$ Brain is rich in oxidizable lipid. Lipid oxidative damage causes loss to brain cells irreversibly, leading to brain cells dysfunction. ${ }^{20)}$ In this study MDA content in the hippocampus of the mice in the Scoptreated group significantly increased while SOD activity decreased. The ratio of brain to body weight, especially the ratio of hippocampus to body weight was significantly reduced. The activities of AChE and total NOS decreased and the abilities of learning and memory diminished, suggesting that oxidative stress was closely related to hippocampus neurons degeneration. Treated with Mag, the condition of oxidative stress in mice's hippocampus was improved (SOD activity increased while MDA content decreased). The activities of AChE and total NOS recovered in the hippocampus and the abilities of learning and memory of the mice were improved, indicating that the Mag was able to scavenge oxygen free radicals and increase the oxidation resisting capacity of the hippocampus, thus preventing hippocampus neurons from lipid peroxidation damage. It may be one of the mechanisms of the reversal of hippocampus neurons degeneration.

Recent research showed, donepezil, considered as one of the most effective drug for the treatment of $\mathrm{AD}$, has the effect to delay or prevent cholinergic neuronal degeneration that is partially associated with the increasing blood flow of the damaged brain tissue. It is also through its anti-oxidation effect that donepezil protects hippocampus neurons from free radicals damage, thereby significantly improves learning and memory impairment of AD model rats. ${ }^{16)}$ These are consistent with the results of this study.

This study demonstrates that the effect of Mag to restore the activities of AChE and total NOS in the hippocampus in a dose-dependent manner is superior to that of donepezil. Therefore it is likely that the improving effects of Mag on the abilities of learning and memory may be, at least in part, mediated by maintenance of density of AChE positive nerve fibers in hippocampus, AChE and total NOS activities.

Taken together, our results clearly showed that Mag ameliorates learning and memory impairment induced by Scop. The underlying mechanisms may be associated with the antioxidative effects of Mag and its protective effects on hippocampus cholinergic neurons.

Acknowledgments This study was supported by the Grant for the construction of the technique plate for evaluation of the pharmacodynamics of new drugs in Xinjiang from the Department of Science and Technology of Xinjiang province 201233150. This work was also supported by the grant from the Department of Science and Technology of Guangdong province to Song Z (No. 2009B030801163). We are grateful to Professor Haihe Wang from Department of Biochemisty, Zhongshan School of Medicine, Sun Yet-Sen University for the experiment suggestions, assistances and the critical read- ing of this manuscript.

\section{REFERENCES}

1) Walsh DM, Selkoe DJ. Deciphering the molecular basis of memory failure in Alzheimer's disease. Neuron, 44, 181-193 (2004).

2) Bigl V, Arendt T. Cholinergic neurons of the central nervous system: morphofunctional aspects. Acta Psychiatr. Scand. Suppl., 366 (S366), 7-13 (1991).

3) Mohapel P, Leanza G, Kokaia M, Lindvall O. Forebrain acetylcholine regulates adult hippocampal neurogenesis and learning. Neurobiol. Aging, 26, 939-946 (2005).

4) Gökçek-Saraç Ç, Karakurt S, Adalı O, Jakubowska-Doğru E. Correlation between hippocampal levels of neural, epithelial and inducible NOS and spatial learning skills in rats. Behav. Brain Res., 235, 326-333 (2012).

5) Frölich L. The cholinergic pathology in Alzheimer's disease-Discrepancies between clinical experience and pathophysiological findings. J. Neural Transm., 109, 1003-1013 (2002).

6) Fibiger HC. Cholinergic mechanisms in learning, memory and dementia: a review of recent evidence. Trends Neurosci., 14, 220-223 (1991).

7) Collerton D. Cholinergic function and intellectual decline in Alzheimer's disease. Neuroscience, 19, 1-28 (1986).

8) Chen YL, Lin KF, Shiao MS, Chen YT, Hong CY, Lin SJ. Magnolol, a potent antioxidant from Magnolia officinalis, attenuates intimal thickening and MCP-1 expression after balloon injury of the aorta in cholesterol-fed rabbits. Basic Res. Cardiol., 96, 353-363 (2001).

9) Shih $\mathrm{HC}$, Wei $\mathrm{YH}$, Lee $\mathrm{CH}$. Magnolol alters the course of endotoxin tolerance and provides early protection against endotoxin challenge following sublethal hemorrhage in rats. Shock, 22, 358-363 (2004).

10) Matsui N, Takahashi K, Takeichi M, Kuroshita T, Noguchi K, Yamazaki K, Tagashira H, Tsutsui K, Okada H, Kido Y, Yasui Y, Fukuishi N, Fukuyama Y, Akagi M. Magnolol and honokiol prevent learning and memory impairment and cholinergic deficit in SAMP8 mice. Brain Res., 1305, 108-117 (2009).

11) Hou YC, Chao PD, Chen SY. Honokiol and magnolol increased hippocampal acetylcholine release in freely-moving rats. Am. J. Chin. Med., 28, 379-384 (2000).

12) Khaing Z, Kang D, Camelio AM, Schmidt CE, Siegel D. Hippocampal and cortical neuronal growth mediated by the small molecule natural product clovanemagnolol. Bioorg. Med. Chem. Lett., 21, 4808-4812 (2011).

13) Myhrer T. Neurotransmitter systems involved in learning and memory in the rat: a meta-analysis based on studies of four behavioral tasks. Brain Res. Brain Res. Rev., 41, 268-287 (2003).

14) Morris R. Developments of a water-maze procedure for studying spatial learning in the rat. J. Neurosci. Methods, 11, 47-60 (1984).

15) Schliebs R, Arendt T. The significance of the cholinergic system in the brain during aging and in Alzheimer's disease. J. Neural Transm., 113, 1625-1644 (2006).

16) Hatip-Al-Khatib I, Takashi A, Egashira N, Iwasaki K, Fujiwara M. Comparison of the effect of TAK-147 (zanapezil) and E-2020 (donepezil) on extracellular acetylcholine level and blood flow in the ventral hippocampus of freely moving rats. Brain Res., 1021, 169-176 (2004).

17) Georgiadou G, Pitsikas N. Repeated administration of the nitric oxide synthase inhibitor L-NAME differentially affects rats' recognition memory. Behav. Brain Res., 224, 140-144 (2011).

18) Rushaidhi M, Collie ND, Zhang H, Liu P. Agmatine selectively improves behavioural function in aged male Sprague-Dawley rats. Neuroscience, 218, 206-215 (2012).

19) Baluchnejadmojarad T, Roghani M. Chronic epigallocatechin-3-gallate ameliorates learning and memory deficits in diabetic rats via modulation of nitric oxide and oxidative stress. Behav. Brain Res., 
224, 305-310 (2011).

20) Loo DT, Copani A, Pike CJ, Whittemore ER, Walencewicz AJ, Cotman CW. Apoptosis is induced by $\beta$-amyloid in cultured central nervous system neurons. Proc. Natl. Acad. Sci. U.S.A., 90, 79517955 (1993).

21) Boissiere F, Faucheux B, Ruberg M, Agid Y, Hirsch EC. Decreased TrkA gene expression in cholinergic neurons of the striatum and basal forebrain of patients with Alzheimer's disease. Exp. Neurol., 145, 245-252 (1997).
22) Mufson EJ, Ikonomovic MD, Styren SD, Counts SE, Wuu J, Leurgans S, Bennett DA, Cochran EJ, DeKosky ST. Preservation of brain nerve growth factor in mild cognitive impairment and Alzheimer disease. Arch. Neurol., 60, 1143-1148 (2003).

23) Shen ZX. CSF cholinesterase activity in demented and non-demented subjects. Neuroreport, 9, 483-488 (1998).

24) Knox LT, Jing Y, Fleete MS, Collie ND, Zhang H, Liu P. Scopolamine impairs behavioural function and arginine metabolism in the rat dentate gyrus. Neuropharmacology, 61, 1452-1462 (2011). 